Bobby Gaspar at University College London and their colleagues began clinical trials treating children with two forms of severe combined immunodeficiency disease (SCID). The researchers took bone marrow stem cells from each patient, used a viral vector to insert a functioning copy of the defective, diseasecausing gene and then infused the cells back into the patients. In one trial involving 10 children with the 'X-linked' form of SCID, all saw their T-cell immunity recover and all have survived, with the longest follow-up of more than a decade. One child developed leukaemia but is now in remission.

The form of SCID treated in the second trial is caused by a deficiency of an enzyme called adenosine deaminase. Four of the six children in this trial had their immunity restored and two even cleared a persistent viral infection.

Sci. Transl. Med. 3, 97ra79;

97ra80 (2011)

For a longer story on this research, see go.nature.com/ q8xyrj

\section{ENVIRONMENTAL RADIATION}

\section{Where the}

\section{radiation went}

Most of the radiation emitted by Japan's Fukushima Daiichi nuclear power plant after March's earthquake was blown out to sea, but some was deposited at hot spots on land.

Toshimasa Ohara and his colleagues at the National Institute for Environmental Studies in Tsukuba, Japan, simulated the transport and deposition of iodine-131 and caesium-137, two radioactive materials that were released in large quantities. They found that $13 \%$ of iodine- 131 and $22 \%$ of caesium- 137 fell on land. Surprisingly, the deposition created hot spots at which caesium-137 levels reached up to 20 kilobecquerels per square metre as far away as the northern part of the Kanto Plain, some 250 kilometres

from the plant.

The levels predicted by the model roughly matched those recorded at 15 monitoring stations in the surrounding prefectures.

Geophys. Res. Lett. http://dx.doi. org/10.1029/2011GL048689 (2011)

\title{
SYNTHETIC BIOLOGY
}

\section{Bacteria engineered to kill}

An engineered strain of the bacterium Escherichia coli can sense the presence of the opportunistic human pathogen Pseudomonas aeruginosa and produce a toxin to kill it.

Chueh Loo Poh, Matthew

Wook Chang and their colleagues at the Nanyang Technological

University

in Singapore

engineered E. coli
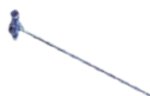

to make a protein that

binds to signalling molecules released by $P$. aeruginosa. The resulting complex activates two gene systems that the authors had introduced into E. coli: one creates the toxin pyocin, which kills $P$. aeruginosa; the other generates a protein that causes E. coli cells to burst, releasing the toxin.

When these E. coli were cultured together with P. aeruginosa, they killed $99 \%$ of the target bacteria.

Mol. Syst. Biol. 7, 521 (2011)

\section{ENERGY TECHNOLOGY}

\section{Supersizing a supercapacitor}

Storing much more charge than standard capacitors, supercapacitors are used in batteries for consumer electronics. Now researchers at the National University of Singapore have devised a simple membrane-based supercapacitor that they say will be easier to scale up than the current alternatives.

Xian Ning Xie and his colleagues used a polystyrenebased polymer to deposit a soft, foldable membrane that, when sandwiched between and

COMMUNITY CHOICE

The most viewed papers in science

\section{A story of chromosome number}

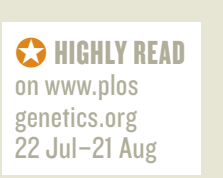

Tracing 150 million years of evolution in the yeast genome has revealed how the microorganism's chromosome numbers have waxed and waned over time.

Genomic upheaval hit about 100 million years ago, when the genome of a yeast ancestor duplicated, doubling the number of chromosomes from 8 to 16 . Today, the chromosome count in various species ranges from 6 to 16 .

Jonathan Gordon and his colleagues at Trinity College Dublin in Ireland studied several yeast species - some of which arose before the genome duplication event and others after - that have different numbers of chromosomes and last shared a common ancestor about 150 million years ago. The authors focused on two important structures: telomeres, which cap the ends of chromosomes, and centromeres, which help to direct chromosomes into dividing cells. They found that, in most cases, yeast species downsizing their complement of chromosomes did so by fusing the telomeres of two chromosomes and losing one of the centromeres.

$$
\text { PLoS Genet. 7, e1002190 (2011) }
$$

charged

by two metal

plates, could store charge at 0.2 farads per square centimetre. This is well above the typical upper limit of 1 microfarad per square centimetre for a standard capacitor. The authors tested membranes up to 9 square centimetres in size and found that their charge storage increased linearly with size. Energy Environ. Sci. http:// dx.doi.org/10.1039/c1ee01841h (2011)

\section{ASTROPHYSICS}

\section{'Pioneer anomaly' not so anomalous}

In 1998, scientists poring over trajectory data from the Pioneer 10 (pictured) and 11 spacecraft found an unexplained acceleration acting on the two vessels, which were launched by NASA in the early 1970 s to observe Jupiter. This led theorists to invoke exotic new $\frac{5}{2}$ theories of gravity. But by rescuing and analysing additional data from decadesold archives, Slava Turyshev at NASA's Jet Propulsion Laboratory in Pasadena, California, and his colleagues say that standard physics can explain what is known as the 'Pioneer anomaly'. The authors boosted the number of tracking points for the spacecraft from 30,671 to 122,591 and found that the acceleration, instead of being constant, was actually slowly decreasing with time. This would be consistent with the emission of heat from a degrading radioisotope power source on board each probe, which would apply a tiny amount of force to the spacecraft.

Phys. Rev. Lett. 107, 081103 (2011)

\section{$\rightarrow$ NATURE.COM}

For the latest research published by Naturevisit:

www.nature.com/latestresearch 